\title{
Effects of High Stimulation Current on the Induction of Ventricular Tachycardia
}

\author{
FRED MORADY, MD, LORENZO A. DICARLO, Jr., MD, L. BING LIEM, DO, \\ RYSZARD B. KROL, MD, and JEFFREY M. BAERMAN, MD
}

\begin{abstract}
Programmed stimulation at 2 right ventricular sites with 1 to 3 extrastimuli was performed at current strengths of twice diastolic threshold (1.0 $\pm 0.2 \mathrm{~mA}$, mean \pm standard deviation) and $10 \mathrm{~mA}$ in $41 \mathrm{pa}$ tients undergolng an electrophysiologic study because of sustained ventricular tachycardia (VT) (11 patients), nonsustained VT (19 patients) or unexplained syncope (11 patients). In 26 patients, VT was not induced by programmed stimulation at twice diastolic threshold. Programmed stimulation at 10 $\mathrm{mA}$ induced VT or ventricular fibrillation in 16 of these 26 patients $(62 \%)$. In 4 of 16 patients, the coupling intervals of the extrastimuli that induced $\mathrm{VT} /$ ventricular fibrillation at $10 \mathrm{~mA}$ were all equal to or longer than the shortest coupling intervals resulting in ventricular capture at twice diastolic threshold. Fifteen patients had inducible VT at twice diastolic threshold. Programmed stimulation at 10 mA induced a similar VT in 12 of these patients, but resulted in no VT induction in 3 of 15 patients $(20 \%)$, despite ventricular capture at the same coupling
\end{abstract}

intervals that had induced VT at twice diastolic threshold.

This study shows that programmed stimulation at a high current strength may either facilitate or prevent induction of VT. Facilitation of VT induction usually is attributable to a shortening of ventricular refractoriness and the ability of extrastimuli at $\mathbf{1 0}$ $m A$ to capture the ventricle at shorter coupling intervals than possible at twice diastolic threshold. However, in $25 \%$ of cases, the facilitation of VT induction by $10-\mathrm{mA}$ stimuli is not explained by a shortening of ventricular refractoriness. In these cases, and in the patients in whom 10-mA stimuli prevent the induction of VT that was inducible at twice diastolic threshold, the effects of high current strength appear to be mediated through some other mechanism. Other possible mechanisms include an effect on temporal dispersion of refractoriness or on the pattern or extent of ventricular activation.

(Am J Cardiol 1985;56:73-78)
Programmed ventricular stimulation is most frequently performed with a stimulus current strength 2 times the diastolic excitability threshold. However, a wide variety of other current strengths has also been used in some electrophysiology laboratories, ranging from 4 times the diastolic threshold to $20 \mathrm{~mA} \cdot{ }^{1-5}$ Some reports indicate that programmed stimulation with current strengths greater than twice diastolic threshold result in an increased yield of clinically meaningful ventricular $\operatorname{arrhythmias}^{4,5}$; others indicate that high stimulation current simply increased the yield of nonclinical

From the Division of Cardiology, Department of Internal Medicine, University Hospitals, University of Michigan Medical Center, Ann Arbor, Michigan. Manuscript received December 31, 1984; revised manuscript received March 4, 1985.

Address for reprints: Fred Morady, MD, Division of Cardiology, Room W-11511, University Hospitals, 1405 East Ann Street, Ann Arbor, Michigan 48109. arrhythmias rather than those that are clinically significant. 6,7

Aside from the issue of whether the induced arrhythmia is clinically meaningful, it is nevertheless clear that a high stimulation current more often results in the induction of ventricular tachycardia (VT) than does a current strength that is twice diastolic threshold. However, the mechanism by which a high stimulation current affects the results of programmed ventricular stimulation is not clear. Although prior studies have investigated the effects of graded stimulation currents on the induction of VT or ventricular fibrillation (VF) in animals, 8,9 the mechanism by which an increased stimulus strength affects the induction of VT in humans has not been investigated in a systematic fashion.

This prospective study compares the effects of high and low current strength on the induction of $\mathrm{VT}$ in an attempt to clarify how an increase in the stimulus cur- 
rent strength affects the results of programmed ventricular stimulation.

\section{Methods}

The subjects in this prospective study included 41 patients who were referred to the University of Michigan Medical Center for an electrophysiologic study because of sustained VT (11 patients), nonsustained VT (19 patients) or unexplained syncope (11 patients). There were 29 men and 15 women, mean age $53 \pm 16$ years. Nineteen patients had coronary artery disease and a history of a myocardial infarction, 12 had an idiopathic dilated cardiomyopathy, and 10 had no identifiable structural heart disease.

After the patients gave informed consent, they underwent an electrophysiologic study in the fasting, unsedated state, at least 4 half-lives after discontinuation of antiarrhythmic drugs. Twelve patients were being treated with digoxin, which was not discontinued. Two quadripolar electrode catheters were inserted percutaneously into a femoral vein and positioned in the appropriate cardiac chamber. After the completion of atrial stimulation whenever clinically indicated, the electrode catheters were positioned against the apex and outflow tract of the right ventricle. Electrocardiographic leads $\mathrm{V}_{1}$, I and III, and intracardiac right ventricular electrograms were displayed on an oscilloscope and recorded at a paper speed of 25 to $100 \mathrm{~mm} / \mathrm{s}$ with an Electronics for Medicine VR 12 recorder. Pacing was performed with a programmable stimulator (Bloom Associates). The slimuli had a pulse width of $2 \mathrm{~ms}$.

Sustained VT was defined as VT lasting 30 seconds or longer or requiring direct-current countershock or overdrive pacing for termination. Nonsustained VT was defined as VT at least 4 beats in duration, terminating spontaneously within 30 seconds.

The following stimulation protocol was used. Stimulation was initially performed with a current strength that was 2 times the diastolic stimulation threshold. The mean current strength was $1.0 \pm 0.2 \mathrm{~mA}$ (range 0.6 to 1.5 ). Using a drive train of 6 to 8 stimuli at a cycle length of 600 or $500 \mathrm{~ms}$, programmed stimulation was performed with a single extrastimulus $\left(\mathrm{S}_{2}\right)$, then double extrastimuli $\left(\mathrm{S}_{2} \mathrm{~S}_{3}\right)$ at the right ventricular apex. This was repeated using a drive cycle length of $400 \mathrm{~ms}$. Single and double extrastimuli were then introduced at the right ventricular outflow tract using the same 2 drive cycle lengths that were used at the right ventricular apex. Programmed stimulation was then performed using 2 drive cycle lengths at the right ventricular apex with triple extrastimuli $\left(\mathrm{S}_{2} \mathrm{~S}_{3} \mathrm{~S}_{4}\right)$ with $S_{2}$ and $S_{3}$ initially positioned $30 \mathrm{~ms}$ beyond their respective points of ventricular refractoriness, and $\mathrm{S}_{4}$ positioned $300 \mathrm{~ms}$ beyond $S_{3}$. Diastole was scanned with $S_{2} S_{3} S_{4}$ in $10-\mathrm{ms}$ steps. Triple extrastimuli were then introduced at the right ventricular outflow tract. The endpoint for the stimulation protocol performed at a current strength of twice diastolic threshold was either 1 induction of VT or VF requiring direct-current countershock for termination, or 2 or more inductions of sustained VT not requiring direct-current countershock.

After completion of programmed stimulation at a current strength of twice diastolic threshold, the current strength was increased to $10 \mathrm{~mA}$ and the stimulation protocol previously described was repeated without changing the positions of the catheters. Because of time constraints, in some patients, programmed stimulation at $10 \mathrm{~mA}$ was performed at only 1 right ventricular site. The endpoints for the stimulation protocol at a current strength of $10 \mathrm{~mA}$ included the same endpoints used with a current strength of twice diastolic threshold; in addition, to minimize the need for direct-current countershock, the stimulation protocol at $10 \mathrm{~mA}$ was discontinued after 2 inductions of nonsustained polymorphic VT longer than 3 seconds in duration.

Whenever 1 of the arrhythmia endpoints was reached during stimulation at $10 \mathrm{~mA}$, the coupling intervals of the extrastimuli that resulted in VT induction were held constant, and stimulation was repeated after the current strength was decreased back to twice diastolic threshold.

\section{Results}

Programmed stimulation at a current strength of $10 \mathrm{~mA}$ in patients without inducible ventricular tachycardia at twice diastolic threshold: In 26 patients, VT was not induced by programmed stimulation at a current strength of twice diastolic threshold. Programmed stimulation at a current strength of $10 \mathrm{~mA}$ resulted in the induction of nonsustained polymorphic VT in 6 patients (23\%), sustained polymorphic V'T in 2 patients (8\%), sustained unimorphic VT in 3 patients (12\%), and VF in 5 patients (19\%) (Table I). Among these 16 patients in whom VT or VF was induced only by stimulation at a current strength of $10 \mathrm{~mA}$, double extrastimuli were required in 3 patients and triple extrastimuli in 13.

In 4 patients (nos. 1 to 4 ), the coupling intervals of the extrastimuli that induced VT or VF at a current strength of $10 \mathrm{~mA}$ were all either equal to or longer than the shortest coupling intervals that resulted in ventricular capture at a current strength of twice diastolic threshold (Table I). After VT or VF was induced at a current strength of $10 \mathrm{~mA}$, stimulation with the same number of extrastimuli and the same coupling intervals at twice diastolic threshold resulted in ventricular capture without the induction of VT or VF in 3 of the 4 patients (Fig. 1). In 1 of the 4 patients (no. 4), when the current strength was lowered to twice diastolic threshold, the extrastimuli did not all capture the ventricle.

In 12 patients (nos. 5 to 16), the coupling intervals of the extrastimuli that induced VT or VF at a current strength of $10 \mathrm{~mA}$ were shorter than the shortest coupling intervals that had resulted in ventricular capture during initial stimulation at twice diastolic threshold (Table I). A return to twice diastolic threshold with the same coupling intervals that had induced VT at a current strength of $10 \mathrm{~mA}$ resulted in incomplete ventricular capture, and consequently no induced VT, in 10 patients. However, in 2 patients (nos. 15 and 16), extrastimuli at coupling intervals that had initially failed to completely capture the ventricle did so after stimulation at $10 \mathrm{~mA}$ had induced VT. In these 2 patients, repeat stimulation at twice diastolic threshold with coupling intervals identical to those that had induced VT at $10 \mathrm{~mA}$ resulted in the induction of VT in $1 \mathrm{pa}-$ tient, but not the other.

Programmed stimulation at a current strength of 10 $\mathrm{mA}$ failed to induce VT in 10 patients who did not have inducible VT at twice diastolic threshold. When the 16 patients with inducible VT were compared with the 10 patients without inducible VT at a current strength of $10 \mathrm{~mA}$, there was a higher prevalence of coronary artery disease in the former group ( 8 of 16 vs 1 of 10 , $\mathrm{p}<0.05$ ). 
TABLE I Ventricular Tachycardia Induction at a Current Strength of $10 \mathrm{~mA}$ in Patients Without Inducible Ventricular Tachycardia at Twice Diastolic Threshold

\begin{tabular}{|c|c|c|c|c|c|c|}
\hline Pt & $\begin{array}{c}\text { Heart } \\
\text { Disease }\end{array}$ & $\begin{array}{l}\text { Shortest Cls } \\
\text { at } 2 \text { X DT (ms)* }\end{array}$ & $\begin{array}{l}\text { Cls that Induced } \\
\text { VT at } 10 \mathrm{~mA} \text { (ms) }\end{array}$ & $\begin{array}{l}\text { VT Induced } \\
\text { at } 10 \mathrm{~mA}\end{array}$ & $\begin{array}{c}\text { Capture } \\
\text { at } 2 \times \mathrm{DT}^{\dagger}\end{array}$ & $\begin{array}{l}\text { VT Induced } \\
\text { at } 2 \times \mathrm{DT}^{\dagger}\end{array}$ \\
\hline 1 & $\mathrm{CAD}$ & $230 / 180 / 180$ & $240 / 190 / 200$ & P. 7 sed & + & 0 \\
\hline 2 & CAD & $250 / 210 / 210$ & $260 / 220 / 280$ & $\mathrm{U}, \mathrm{S}$ & $t$ & 0 \\
\hline 3 & IDC & $230 / 160 / 150$ & $230 / 160 / 180$ & VF, S & + & 0 \\
\hline 4 & NSHD & $230 / 190 / 160$ & $230 / 200 / 160$ & $\mathrm{P}, 12 \mathrm{sec}$ & 0 & 0 \\
\hline 5 & $C A D$ & $240 / 220$ & $230 / 160$ & P, 12 sec & 0 & 0 \\
\hline 6 & NSHD & $290 / 230 / 190$ & $260 / 220 / 210$ & P, 24 beats & 0 & 0 \\
\hline 7 & NSHD & $220 / 180 / 160$ & $220 / 160 / 140$ & P, 9 beats & 0 & 0 \\
\hline 8 & $\mathrm{CAD}$ & $270 / 230$ & $270 / 220$ & $\mathrm{P}, \mathrm{S}$ & 0 & 0 \\
\hline 9 & IDC & $290 / 270 / 240$ & $290 / 250 / 230$ & $\cup, S$ & 0 & 0 \\
\hline 10 & $\mathrm{CAD}$ & $280 / 240 / 230$ & $280 / 220 / 220$ & $\vec{U}, \mathrm{~S}$ & 0 & 0 \\
\hline 11 & NSHD & $250 / 180 / 180$ & $240 / 190 / 170$ & VF, S & 0 & 0 \\
\hline 12 & CAD & $220 / 190 / 190$ & $230 / 180 / 170$ & VF, $s$ & 0 & 0 \\
\hline 13 & $\mathrm{CAD}$ & $220 / 170 / 130$ & $240 / 150 / 130$ & VF, S & 0 & 0 \\
\hline 14 & IDC & $230 / 170 / 160$ & $220 / 130 / 130$ & VF, S & 0 & 0 \\
\hline 15 & CAD & $270 / 240$ & $260 / 180$ & $\mathrm{P}, \mathrm{S}$ & + & $P(S)$ \\
\hline 16 & NSHD & $260 / 210 / 190$ & $260 / 190 / 180$ & P, 9 beats & + & 0 \\
\hline
\end{tabular}

* Shortest coupling intervals $\left(S_{1} S_{2} / S_{2} S_{3} / S_{3} S_{4}\right)$ associated with ventricular capture at current strength of twice diastolic threshold, before programmed stimulation at $10 \mathrm{~mA}$. The drive cycle length in each case was the same as that used to induce ventricular tachycardia (VT) at $10 \mathrm{~mA}$.

$\uparrow$ Refers to ventricular capture or VT induction at twice diastolic threshold, after induction of VT/ventricular fibrillation at $10 \mathrm{~mA}$, with the same number of extrastimuli and coupling intervals that induced VT/ventricular fibrillation at $10 \mathrm{~mA}$.

$\mathrm{CAD}=$ coronary artery disease; $\mathrm{Cl}=$ coupling interval; IDC = idiopathic dilated cardiomyopathy; NSHD $=$ no structural heart disease; $P=$ polymorphic; $S=$ sustained; $V F=$ ventricular fibrillation; $V T=$ ventricular tachycardia; $U=$ unimorphic, $2 X \mathrm{DT}=$ twice diastolic threshold; $+=$ yes; $0=$ no.

A.
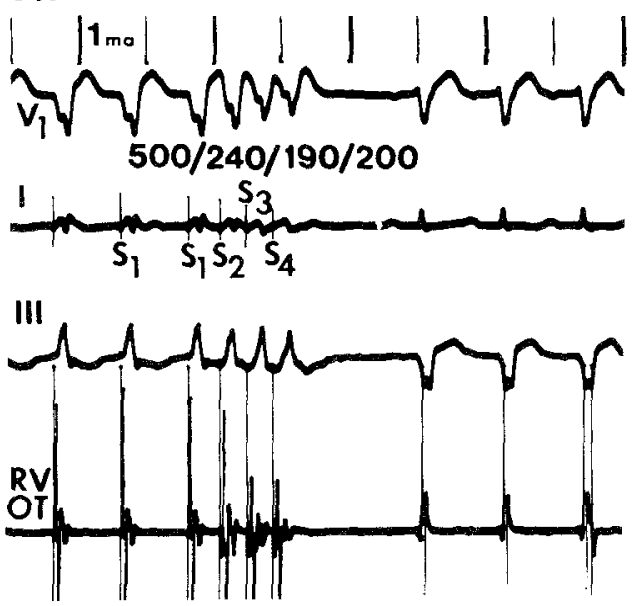

C.
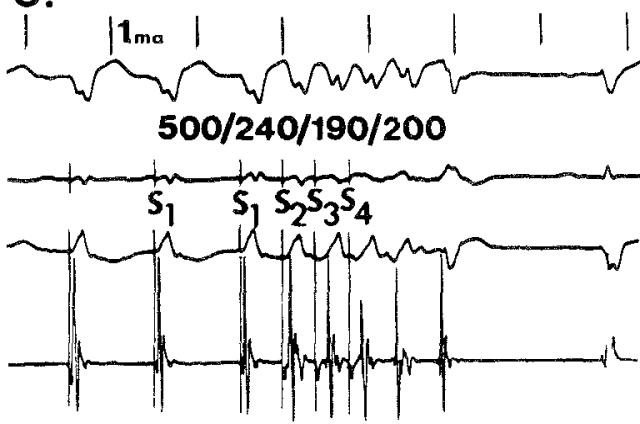

B.
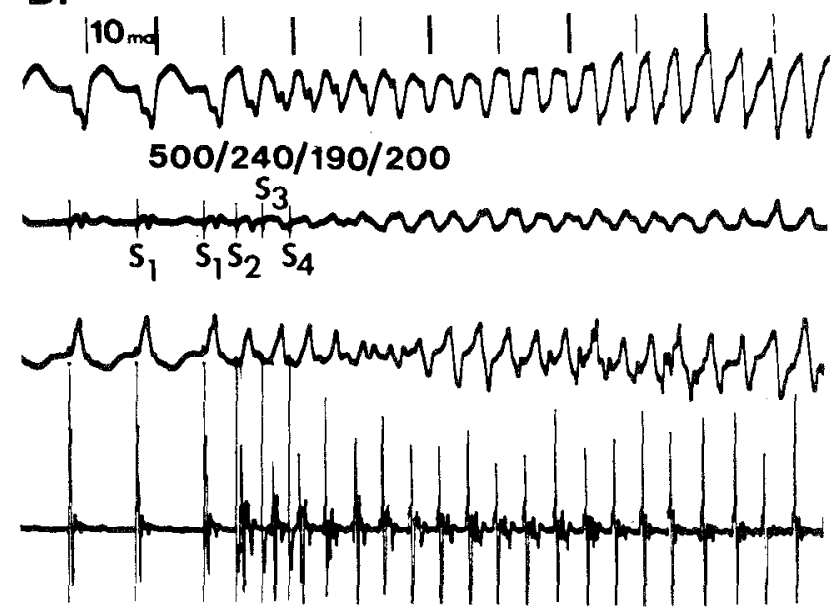

D.

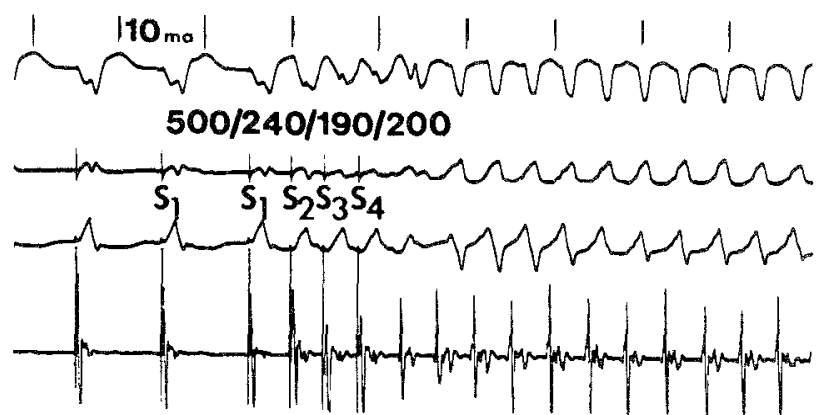

FIGURE 1. Programmed stimulation at the right ventricular outflow tract in patient 1 , at a drive cycle length $\left(S_{1} S_{1}\right)$ of 500 ms, and with $S_{1} S_{2} / S_{2} S_{3} / S_{3} S_{4}$ intervals of $240 / 190 / 200 \mathrm{~ms}$. Top to bottom, leads $V_{1}, I$ and III, and an electrogram recorded at the right ventricular outflow tract (RVOT). Time lines represent $500-\mathrm{ms}$ intervals. A, when the current strength was twice diastolic threshold (1 mA), ventricular tachycardia (VT) was not induced. $\mathbf{B}$, after the current strength was increased to $10 \mathrm{~mA}, 7$ seconds of polymorphic VT (mean cycle length $220 \mathrm{~ms}$ ), was induced. $\mathbf{C}$, when the current strength was decreased back to $1 \mathrm{~mA}$, there was again ventricular capture by all of the extrastimuli, but $V T$ was not induced. $D$, an increase in current strength to $10 \mathrm{~mA}$ again resulted in the induction of nonsustained polymorphic VT lasting 5 seconds. 
TABLE II Ventricular Tachycardia Induction at a Current Strength of $10 \mathrm{~mA}$ in Patients With Inducible Ventricular Tachycardia at Twice Diastolic Threshold

\begin{tabular}{|c|c|c|c|c|c|}
\hline$P t$ & $\begin{array}{c}\text { Heart } \\
\text { Disease }\end{array}$ & $\begin{array}{l}\text { Cls that induced } \\
\text { VT at } 2 \times \text { DT (ms) }\end{array}$ & $\begin{array}{l}\text { VT Induced } \\
\text { at } 2 \times D T\end{array}$ & Cls at $10 \mathrm{~mA}(\mathrm{~ms})^{*}$ & $\begin{array}{l}\text { VT Induced } \\
\text { at } 10 \mathrm{~mA}\end{array}$ \\
\hline $\begin{array}{l}17 \\
18 \\
19 \\
20 \\
21 \\
22 \\
23 \\
24 \\
25 \\
26 \\
27 \\
28 \\
29 \\
30 \\
31\end{array}$ & $\begin{array}{l}\text { NSHD } \\
\text { CAD } \\
\text { CAD } \\
\text { NSHD } \\
\text { CAD } \\
\text { IDC } \\
\text { CAD } \\
\text { CAD } \\
\text { CAD } \\
\text { CAD } \\
\text { IDC } \\
\text { NSHD } \\
\text { CAD } \\
\text { CAD } \\
\text { CAD }\end{array}$ & $\begin{array}{l}250 / 210 / 200^{\dagger} \\
260 / 210 / 210 \\
240 / 200 \\
230 / 180 / 170 \\
220 / 160 / 140 \\
140 / 130 \\
220 / 160 / 150 \\
250 / 190 / 180 \\
240 / 190 \\
260 / 230 \\
260 / 190 / 170 \\
230 \\
290 / 280 \\
270 / 220 / 210 \\
270 / 210 / 180\end{array}$ & $\begin{array}{l}P, 13 \text { beats } \\
\text { RBBB, } S \\
\text { RBBB, } 13 \text { beats } \\
P, 8 \text { sec } \\
P, 9 \text { beats } \\
P, 5 \text { sec } \\
P, 8 \text { beats } \\
\text { P, S } \\
\text { P, S } \\
\text { RBBB, } 20 \text { sec } \\
\text { RBBB, } 12 \text { sec } \\
\text { LBBB, } 11 \text { sec } \\
\text { LBBB, S } \\
\text { LBBB, S } \\
\text { RBBB, S }\end{array}$ & $\begin{array}{l}250 / 190 / 170 \\
260 / 190 / 200 \\
240 / 180 \\
230 / 180 / 170 \\
230 / 180 / 210 \\
200 / 140 / 140 \\
220 / 160 / 150 \\
250 / 180 / 170 \\
240 / 190 \\
260 / 260 \\
260 / 190 / 170 \\
200 \\
290 / 250 \\
280 / 210 / 200 \\
270 / 200 / 170\end{array}$ & $\begin{array}{l}0 \\
0 \\
0 \\
P, S \\
P, 21 \text { beats } \\
P, S \\
P, 8 \text { sec } \\
P, 4 \text { sec } \\
P, 7 \text { sec } \\
\text { RBBB, } 18 \text { sec } \\
\text { RBBB, } 15 \text { sec } \\
\text { LBBB, } 7 \text { sec } \\
\text { LBBB, S } \\
\text { LBBB, S } \\
\text { RBBB, S }\end{array}$ \\
\hline
\end{tabular}

* Coupling intervals that resulted in induction of ventricular tachycardia or when ventricular tachycardia was not induced were the shortest coupling intervals associated with ventricular capture.

$\dagger S_{1} S_{2} / S_{2} S_{3} / S_{3} S_{4}$ intervals in milliseconds.

$\mathrm{LBBB}=$ left bundle branch block configuration; RBBB = right bundle branch block configuration; other abbreviations as in Table 1 .

Programmed stimulation at a current strength of $10 \mathrm{~mA}$ in patients with inducible ventricular tachycardia at twice diastolic threshold: Fifteen patients had inducible VT at a current strength of twice diastolic threshold. The VT was polymorphic in 8 patients and unimorphic in 7 ; it was induced by a single extrastimulus in 1 patient, double extrastimuli in 2 patients, and triple extrastimuli in 12 patients. Programmed stimulation at a current strength of $10 \mathrm{~mA}$ also resulted in the induction of VT in 12 of these 15 patients (Table II). However, in 3 patients who had inducible VT at a current strength of twice diastolic threshold, VT was not induced by programmed stimulation with up to 3 extrastimuli at a current strength of $10 \mathrm{~mA}$ (Fig. 2). In each of these 3 patients, the shortest coupling intervals associated with ventricular capture at $10 \mathrm{~mA}$ were all equal to or shorter than the coupling intervals that had resulted in VT induction at twice diastolic threshold.

Among the other 12 patients with inducible VT at twice diastolic threshold, programmed stimulation at $10 \mathrm{~mA}$ induced VT that was similar in configuration and cycle length, with the same number of extrastimuli and similar coupling intervals.

\section{Discussion}

The results of this study indicate that programmed stimulation with a high-current strength $(10 \mathrm{~mA})$ induces VT or VF in a large proportion (52\%) of patients who do not have inducible VT or VT at a current strength of twice diastolic threshold. In many patients (75\%), this discordant response to programmed ventricular stimulation may be explained by the ability of extrastimuli at $10 \mathrm{~mA}$ to capture the ventricle at shorter coupling intervals than is possible when the current strength is twice diastolic threshold.

In accord with the above-mentioned finding are the results of prior experimental studies. ${ }^{8,9}$ Michelson et al $^{8}$ reported that the use of increased current strength facilitated the induction of VT in a chronic canine model of myocardial infarction by its effects on ventricular refractoriness; the induction of VT by pacing stimuli of high-current strength was always attributable to ventricular capture at coupling intervals that could not be attained when the current strength was twice diastolic threshold. Also, Hamer et $\mathrm{al}^{9}$ reported that the ability to capture the ventricle at shorter coupling intervals was almost always the mechanism by which pacing stimuli of high-current strength induced VF in the normal canine heart in which VF could not be induced when the stimuli were twice diastolic threshold.

In $25 \%$ of patients in this study in whom VT or VF was induced by extrastimuli of $10 \mathrm{~mA}$ but not twice diastolic threshold, the discordant response was not attributable to a difference in coupling intervals. This observation suggests that at least in some patients, a high current strength facilitates the induction of ventricular arrhythmias by a mechanism other than simply allowing extrastimuli to capture the ventricle at shorter coupling intervals. At least 2 other mechanisms are possible. First, a high current strength may facilitate the initiation of VT or VF by an effect on recovery of excitability. In the open-chest dog, Spear et $\mathrm{al}^{10}$ showed that an increase in current intensity increased the temporal dispersion in recovery of excitability in the myocardium surrounding the stimulation site, thereby lowering the VF threshold. A second mechanism by which a highcurrent strength may facilitate the induction of VT or VF is by depolarizing more myocardial cells than does a stimulus of twice diastolic threshold. This might alter the pattern of ventricular activation in such a way as to favor the initiation of reentry, or might allow for depolarization of a region of myocardium containing a potential substrate for reentry.

Although a current strength of $10 \mathrm{~mA}$ usually facilitated the induction of VT, the opposite was also observed. When the current strength was increased to $10 \mathrm{~mA}$, VT was no longer inducible in $20 \%$ of patients who had inducible VT at twice diastolic threshold, despite ventricular capture at the same coupling intervals 
A.

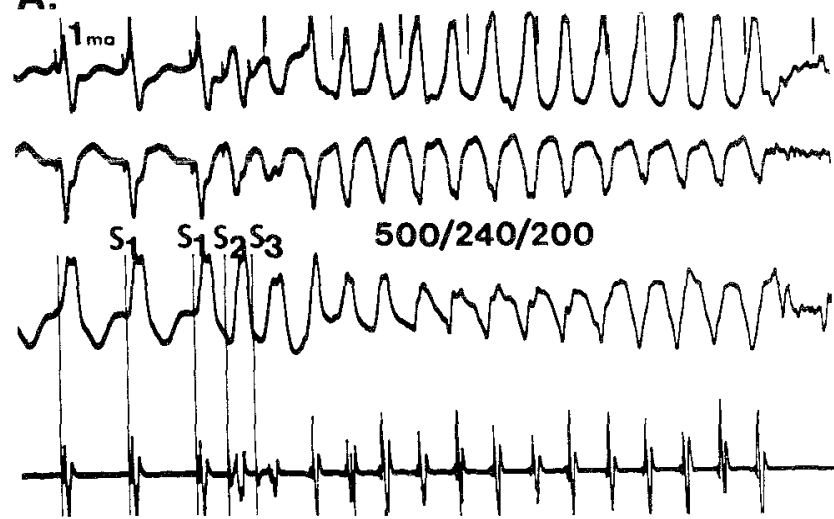

c.
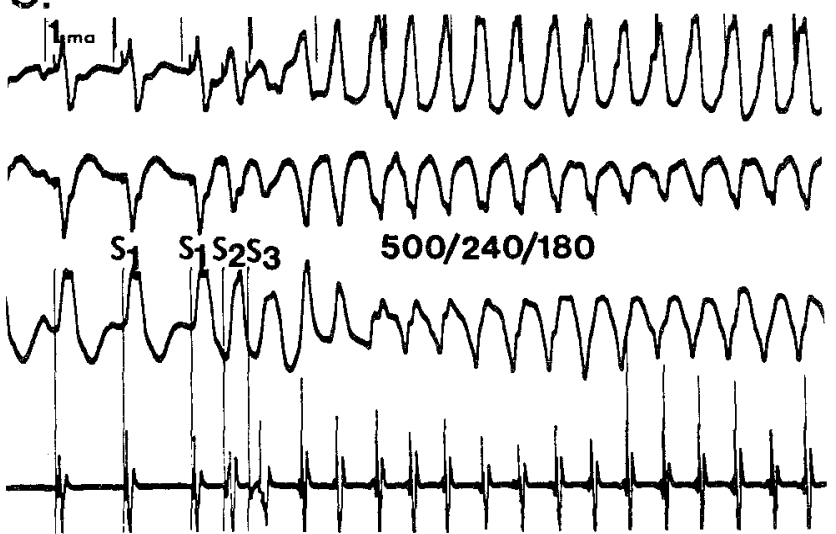

B.

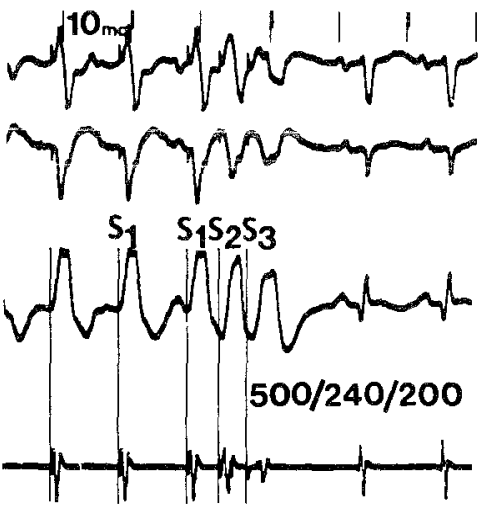

D.
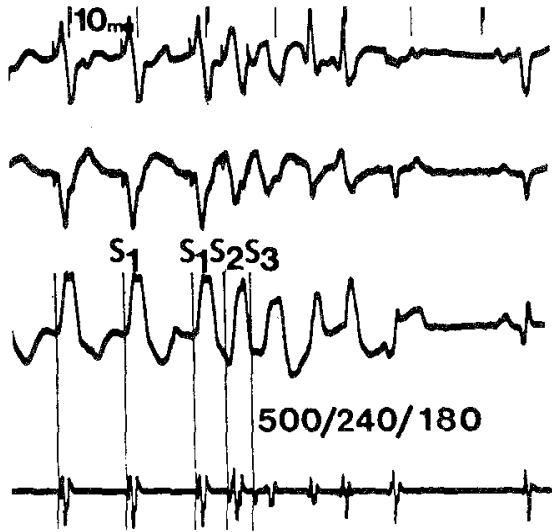

FIGURE 2. Programmed stimulation at the right ventricular outflow tract in patient 17 , at a drive cycle length $\left(S_{1} S_{1}\right)$ of 500 ms. Top to bottom, leads $\mathrm{V}_{1}, \mathrm{l}$, and III, and an electrogram recorded at the right ventricular outflow tract. Time lines represent 500-ms intervals. A, when the current strength was twice diastolic threshold (1 mA) and the $S_{1} S_{2} / S_{2} S_{3}$ intervals were $240 / 200 \mathrm{~ms}$, a 13-beat run of polymorphic ventricular tachycardia (VT) (mean cycle length $270 \mathrm{~ms}$ ), was induced. $\mathbf{B}$, an increase in current strength to $10 \mathrm{~mA}$ resulted in ventricular capture, but VT was not induced. $\mathbf{C}$, at current of $1 \mathrm{~mA}, \mathrm{~S}_{1} \mathrm{~S}_{2} / \mathrm{S}_{2} \mathrm{~S}_{3}$ intervals of $240 / 180 \mathrm{~ms}$ also induced polymorphic VT (24 beats). D, an increase in current strength to $10 \mathrm{~mA}$ again resulted in ventricular capture without the induction of VT.

at both levels of current strength. This observation provides additional evidence that an increase in current strength may affect VT induction in some way other than simply shortening ventricular refractoriness. An effect on temporal dispersion in recovery of excitability or on the pattern of ventricular activation, while facilitating the induction of reentry in some patients, may also prevent the initiation of reentry in some patients who have the appropriate substrate for reentry when the current strength is twice diastolic threshold. Brugada et al, ${ }^{7}$ in a preliminary report, found that in a group of 20 patients with clinically documented VT, VT was induced by stimulation only at twice diastolic threshold, and not by stimulation at $20 \mathrm{~mA}$, in 9 patients $(45 \%)$.

In 2 patients (nos. 15 and 16), stimuli at twice diastolic threshold captured the ventricle at the same coupling intervals that induced VT at $10 \mathrm{~mA}$, but only after VT had been induced by the $10 \mathrm{~mA}$ stimuli. The explanation for this phenomenon is unclear; one possibility is that the induction of VT by $10-\mathrm{mA}$ stimuli results in the release of catecholamines that shorten ventricular refractoriness, thereby enabling stimuli at twice diastolic threshold to capture the ventricle. In patient 15 the extrastimuli at twice diastolic threshold did subsequently induce sustained polymorphic VT, similar to the V'T that had been induced by stimulation at $10 \mathrm{~mA}$. In this patient V'T induction clearly was dependent only on ventricular capture at critical coupling intervals and not on the stimulus current strength per se. However, in patient 16, extrastimuli at twice diastolic threshold did not induce VT even after ventricular capture became possible at the same coupling intervals that resulted in VT induction at $10 \mathrm{~mA}$. This result indicates that when high current strength facilitates the induction of VT at coupling intervals that did not cap-. ture the ventricle at twice diastolic threshold, the possibility is not ruled out that a mechanism other than shortening of ventricular refractoriness is responsible for the induction of VT.

Among the patients without inducible VT at twice diastolic threshold, the only discernible difference between the patients in whom a current strength of $10 \mathrm{~mA}$ did and those in whom it did not facilitate VT induction was that there was a higher prevalence of coronary artery disease in the group that did have inducible VT at 10 $\mathrm{mA}$. This finding suggests that the facilitation of VT induction by the use of a high stimulus current strength may be more common in patients who have coronary artery disease. This possibility is consistent with the finding that coronary occlusion in the dog augments the 
increase in temporal dispersion in recovery of excitability that occurs as the stimulus current strength increases. ${ }^{10}$

When programmed stimulation with a current strength of both twice diastolic threshold and $10 \mathrm{~mA}$ induced VT, the configuration of the VT was similar at both current strengths, as were the number of extrastimuli and the coupling intervals that induced the $V^{r} \Gamma$. Unimorphic VT was induced in patients with documented unimorphic VT and was thought to be a clinically meaningful response. However, the polymorphic VT was induced either in patients with documented unimorphic V'I' or in those with unexplained syncope, and was believed to be a laboratory artifact rather than a clinically significant arrhythmia. Among the patients in whom polymorphic VT was induced at a current strength of twice diastolic threshold, an increase in the current strength to $10 \mathrm{~mA}$ never resulted in the induction of unimorphic VT. Furthermore, in only 3 of 16 patients who had an inducible arrhythmia at $10 \mathrm{~mA}$ and not twice diastolic threshold was the induced arrhythmia a unimorphic VT. Although this study was not intended to address the issue of relative sensitivity and specificity of stimulation protocols with high and low current strength, these observations suggest that the use of a stimulus current strength of $10 \mathrm{~mA}$ may compromise the specificity of stimulation protocols to a much greater degree than it enhances sensitivity. Preliminary reports on the sensitivity and specificity of programmed ventricular stimulation protocols using current strengths of 10 or $20 \mathrm{~mA}$ have found this to be the case. 6,7

The principal limitation of the present study is the inability to guarantee the reproducibility of all induced arrhythmias. Arrhythmias requiring direct-current countershock were induced only once at a given current strength to avoid subjecting patients to multiple shocks. Therefore, a discordant response to stimulation at twice diastolic threshold and $10 \mathrm{~mA}$ may be attributable to nonreproducibility of arrhythmia induction instead of a change in the stimulus current strength. To minimize the likelihood of this possibility, discordant responses to stimulation at the high and low current strengths were confirmed whenever feasible by immediately alternating between the 2 current strengths.

In conclusion, this study has demonstrated that programmed stimulation at a high current strength may either facilitate or prevent the induction of VT. Facilitation of VT induction is most often attributable to a shortening of ventricular refractoriness and the ability of extrastimuli at $10 \mathrm{~mA}$ to capture the ventricle at shorter coupling intervals than is possible at twice diastolic threshold. However, in at least $25 \%$ of cases, the facilitation of V'T induction by $10 \mathrm{~mA}$ stimuli cannot be explained by a shortening of ventricular refractoriness. In these cases, and in the patients in whom $10-\mathrm{mA}$ stimuli prevented the induction of VT that was inducible at twice diastolic threshold, the effects of high current strength appear to be mediated through some other mechanism. Other possible mechanisms include an effect on temporal dispersion of refractoriness or on the pattern or extent of ventricular activation.

A clinical implication of this study is that programmed ventricular stimulation at a high current strength should always be preceded by stimulation at twice diastolic threshold. The immediate use of pacing stimuli of high current strength may not only result in a high incidence of arrhythmias that are not clinically meaningful, but also may prevent the induction of VT in some patients who have inducible VT at a current strength of twice diastolic threshold.

\section{References}

1. Waspe LE, Selnfeld D, Ferrick A, Fink D, Scavin G, Wanliss M, Kim SG, Matos JA, Fisher JD. Programmed electrical stimulation to predict late sudden death in survivors of complicated myocardial infarction (abstr). JACC 1984;3:609.

2. DiMarco JP, Garan H, Ruskin JN. Quinidine for ventricular arrhythmias: value of electrophyslologic testing. Am J Cardiol 1983;51:90-95.

3. Bacaner M, Benditt DG. Antiarrhythmic, antifibrillatory, and hemodynamic actions of bethanidine sulfate: an orally effective analog of bretylium for actions of bethanidine sulfate: an orally effective analog of bretylum for
suppression of ventricular tachyarrhythmias. Am J Gardiol 1982;50: suppression $728-734$

4. Hamer A, Vohra J, Hunt D, Sloman G. Prediction of sudden death by electrophysiologic studies in high risk patients surviving acute myocardial Infarction. Am J Cardlol 1982;50:223-229.

5. Richards DA, Cody DV, Denniss AR, Russell PA, Young AA, Uther JB. Ventricular electrical instability: a predictor of death after myocardial infarction. Am J Cardiol 1983;5 1:75-80.

6. Herre JM, Mann DE, Luck JC, Magro SA, Wyndham CR. Effect of third and fourth extrastimuli and increased current on programmed ventricular stimulation-a prospective study (abstr). Circulation 1983;68:suppl III:III243 .

7. Brugada P, Abdollah $\mathbf{H}$, Wellens JHH. Sensitivity of a ventricular stimulation protocol using two ventricular extrastimuli at twice diastolic threshold and $20 \mathrm{~mA}$ (abstr). JACC 1984;3:609.

8. Michelson EL, Spear JF, Moore EN. Strength-interval relations in a chronic canine model of myocardial infarction. Implications for the interpretation of electrophysiologic studies. Circulation 1981;63:1158-1165.

9. Hamer AW, Karagueuzian HS, Sugi K, Zaher CA, Mandel WJ, Peter T. Factors related to the induction of ventricular fibrillation in the normal canine heart by programmed electrical stimulation. JACC 1984;3:751-759.

10. Spear JF, Moore EN, Horowitz LN. Effect of current pulses delivered during the ventricular vulnerable period upon the ventricular fibrillation threshold. Am J Cardiol 1973;32:814-822. 